\title{
Vibration Analysis of Aeroengine Blisk Structure Based on a Prestressed CMS Super-Element Method
}

\author{
Zhijun Li, ${ }^{1}$ Wenjun Yang, ${ }^{2}$ and Huiqun Yuan ${ }^{2,3}$ \\ ${ }^{1}$ School of Resources and Civil Engineering, Northeastern University, Shenyang, China \\ ${ }^{2}$ School of Mechanical Engineering and Automation, Northeastern University, Shenyang, China \\ ${ }^{3}$ College of Science, Northeastern University, Shenyang, China \\ Correspondence should be addressed to Huiqun Yuan; yuan_hq@163.com
}

Received 10 April 2016; Revised 10 August 2016; Accepted 24 August 2016

Academic Editor: Yuri S. Karinski

Copyright (C) 2016 Zhijun Li et al. This is an open access article distributed under the Creative Commons Attribution License, which permits unrestricted use, distribution, and reproduction in any medium, provided the original work is properly cited.

\begin{abstract}
For vibration analysis of aeroengine blisk structure, a prestressed component modal synthesis (CMS) super-element method is put forward with the fixed interface prestressing and free interface super-element approach. Based on this method, natural vibration characteristics of blisk structure are calculated at different modal truncation numbers. Comparing with the accurate result of global method, the selection principle of modal truncation number is obtained which affects the accuracy of prestressed CMS superelement method. Vibration response of two-stage blisk structure is calculated by this method, and the effects of different blade aspect ratios have been discussed on vibration characteristics. The results show that prestressed CMS super-element method is in the high accuracy and efficiency on blisk vibration analysis. Resonant frequencies in vibration response are nearly the same between the first-stage blisk and the second-stage blisk, and they are both approximately located in the range $588 \mathrm{~Hz}-599 \mathrm{~Hz}$. The maximum displacement and dynamic stress are at blade tip and root of the first-stage blisk, respectively. Blade aspect ratio is a key factor of blisk vibration; the effects of blade aspect ratio on natural frequencies are different in the conditions of fixed width and fixed length. This research provides the theoretical basis for dynamic design of aeroengine compressor rotor system.
\end{abstract}

\section{Introduction}

With the increasing development of aeronautical manufacturing technology, a whole system of bladed disk (blisk) has been widely used in newly developed aeroengines. Blade and disk are integrated to the whole system of bladed disk by the advanced technology. Traditional joint of tenon and mortise is removed out between blade and disk; it can greatly simplify the structure to achieve the light-weighting design. Multiple crack failure is avoided in mortise bottom, and the reliability of rotor system is improved significantly.

Many scholars have carried out extensive research on the blisk structure. Ferria et al. [1] developed a blisk numerical model on the flutter stability of subsonic turbine and pointed out the conditions which affected the stability of turbine system. Di Maio and Ewins [2] implemented a practical method to measure vibration response of simplified blisk structure by Scanning Laser Doppler Vibrometer systems. Ji et al. [3] put forward a multidisciplinary optimization method for the design of compressor structure, and this method was verified that it could maintain the harmony and consistency of blisk structure well. Bhaumik et al. [4] conducted a theoretical research on the failure mechanism of turbine blisk; the criteria of avoiding failure were given according to the performance of turbine material. S. Lu and F.-J. Lu [5] presented a weight-lighting optimized design of blisk structure with a guarantee of structural safety. In the field of crack growth, Xu et al. [6] developed some measures to improve the reliability of axial compressor blisk system.

Due to the limitation of experimental conditions and lack of appropriate experimental methods, major approach is the finite element analysis (FEA) in the analysis of structural dynamics. However, for the large complex structures such as aeroengine blade disk system, the number of degrees of freedom (DOFs) can be up to one million in discrete model. Corresponding kinematic equations cannot be solved efficiently, and it is difficult to develop dynamic analysis with 
the finite element method. Although it can be analyzed with FEA, a lot of time would be eventually consumed. Thus, calculation efficiency cannot be guaranteed. From the review of related studies, it can be found that computational method of blisk dynamics remains for further research. So the method of dynamic substructure becomes an appropriate solution method on the basis of modal reduction technology.

Theoretical method and practical application of modal synthesis technology have been researched by related scholars [7-10]. Hurty firstly established the concepts of modal coordinates and modal synthesis method [11], which laid the foundation of fixed interface modal synthesis method. Then, Bampton and Craig Jr. [12] proposed an improved method to make the fixed interface method more simple and practical. This method eliminated the boundary of rigid mode and constraint mode and no longer distinguished between both of them. Hou [13] and Goldman [14] explored the modal synthesis method with free interface. While the effect of higher-order substructure modes is ignored, the accuracy of this method is challenged. MacNeal [15] and Rubin [16] introduced the residual stiffness to consider the effect of higher-order truncated modes; global precision was improved. For further improving Rubin's method, Wang and $\mathrm{Du}$ [17] put forward the dynamic substructure technique with double coordination. In this method, residual stiffness was regarded as the Ritz base; modified free interface method was put into the orbit of Ritz analysis. Comprehensive precision and efficiency were improved greatly. With the reduction technology, Leung [18] concentrated internal coordinates of each substructure to the coordinates of each substructure interface. Motion equation of the system was established according to the conditions of displacement coordination and force equilibrium. Based on Leung's method, Yun et al. [19] developed a super-element modal synthesis method by the frequency conversion of dynamic modes. Although there is a certain progress in the technique of dynamic substructure, its application still needs to be further researched on dynamic analysis.

For the analysis of dynamic characteristics in an aeroengine blisk structure, in our research a prestressed component modal synthesis (CMS) method is proposed with the fixed interface prestressing and free interface superelement approach. Based on this method, natural vibration characteristics of blisk structure are calculated at different modal truncation numbers. Comparing with the accurate result of global method, the selection principle of modal truncation number is obtained which affects the accuracy of prestressed CMS super-element method. Vibration response of two-stage blisk structure is calculated by this method, and the effects of different blade aspect ratios have been discussed on vibration characteristics.

\section{Dynamics Modeling of Aeroengine Blisk Structure}

2.1. The Method of Prestressed CMS Super-Element Method. Blisk structure of aeroengine system is very complex. It is a challenge for numerical simulation, as the number of finite elements is much enormous. In this research, the prestressed CMS super-element method is proposed to analyze dynamic characteristics of blisk structure. By this method, it can solve the structure with large numbers of elements. Moreover, the prestressed CMS super-element method has higher precision and efficiency compared with traditional method.

The prestressed CMS super-element method is based on modal synthesis technology, and it is a method which utilizes matrix reduction technology to reduce model order. For the $i$ th substructure finite element model, the general undamped free vibration equation can be expressed as

$$
M_{i} \ddot{q}_{i}+K_{i} q_{i}=0, \quad(i=1,2, \ldots, n)
$$

where $M_{i}$ is the mass matrix, $K_{i}$ is the stiffness matrix, $q_{i}$ is the displacement vector, and $n$ is the number of substructures.

For components such as blades, they are very thin on the direction of one or two degrees of freedom. At the action of centrifugal force, stress state may affect structural natural frequency and dynamic response. So in the analysis of rotor dynamic characteristics, the effect of centrifugal rigidification should be considered. In our research, the linear stress analysis is developed under the static state; centrifugal load is transformed into structure prestressed matrix $S$. Then, dynamic equation related to centrifugal rigidification can be expressed as

$$
M_{i} \ddot{q}_{i}+K_{i}^{\prime} q_{i}=0, \quad(i=1,2, \ldots, n)
$$

where $K_{i}^{\prime}$ is the stiffness matrix which considers the matrix of prestressed effect. Displacement vector and coefficient matrix in (2) are divided into the master DOF on the boundary and the slaver DOF beyond the boundary. Here subscripting $\mathrm{m}$ denotes the master DOF and subscripting s denotes the slaver DOF. The transformed form is

$$
\begin{aligned}
& {\left[\begin{array}{cc}
M_{\mathrm{mm}} & M_{\mathrm{ms}} \\
M_{\mathrm{sm}} & M_{\mathrm{ss}}
\end{array}\right]_{i}\left\{\begin{array}{l}
\ddot{q}_{\mathrm{m}} \\
\ddot{q}_{\mathrm{s}}
\end{array}\right\}_{i}+\left[\begin{array}{cc}
K_{\mathrm{mm}}^{\prime} & K_{\mathrm{ms}}^{\prime} \\
K_{\mathrm{sm}}^{\prime} & K_{\mathrm{ss}}^{\prime}
\end{array}\right]_{i}\left\{\begin{array}{c}
q_{\mathrm{m}} \\
q_{\mathrm{s}}
\end{array}\right\}_{i}} \\
& \quad=\left\{\begin{array}{l}
0 \\
f_{i}
\end{array}\right\} .
\end{aligned}
$$

In the equation, $f_{i}$ is the interfacial force. Coordinates of substructure are transformed by the following formula:

$$
q_{i}=\Phi_{i}\left\{\begin{array}{c}
p_{\mathrm{m}} \\
p_{\mathrm{s}}
\end{array}\right\}_{i}
$$

Here $p$ is the coordinate of substructure mode, $\Phi_{i}=$ $\left[\begin{array}{cc}E & 0 \\ -K_{\mathrm{ss}}^{\prime-1} K_{\mathrm{sm}}^{\prime} & \Phi_{1}\end{array}\right]_{i}$ is the transformed coordinate matrix in the fixed interface [12], $\Phi_{1}$ is substructure eigenvector in the condition of fixed boundary nodes, and $E$ is the unit matrix. According to formula (4), dynamic equation in formula (2) can be expressed with modal coordinates:

$$
\bar{M}_{i} \ddot{p}_{i}+\bar{K}_{i} p_{i}=0, \quad(i=1,2, \ldots, n)
$$




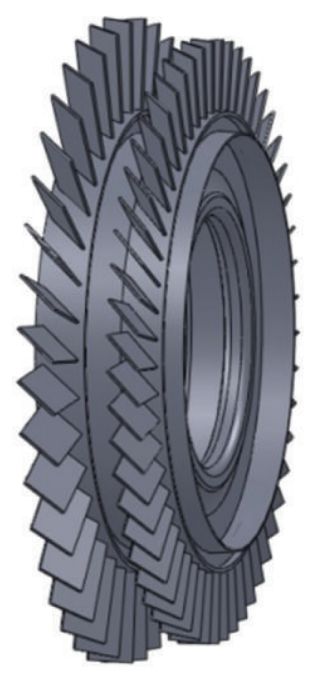

(a)

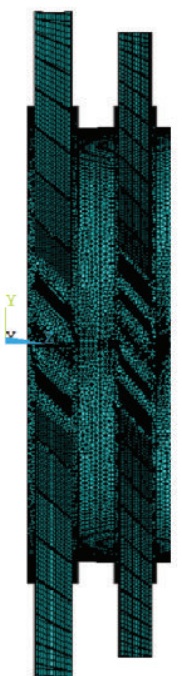

(b)

FIGURE 1: Two-stage blisk structure model: (a) three-dimensional geometry and (b) finite element mesh.

Here $\bar{M}_{i}=\Phi_{i}^{\mathrm{T}} M_{i} \Phi_{i}, \bar{K}_{i}=\Phi_{i}^{\mathrm{T}} K_{i}^{\prime} \Phi_{i}$. Based on the DOF reduction method [11], the following expression can be obtained from formula (4):

$$
q_{i}=\Phi_{i}^{\prime}\left\{\begin{array}{c}
p_{\mathrm{m}} \\
p_{\mathrm{a}}
\end{array}\right\}
$$

In formula (6), $p_{\mathrm{a}}$ is the reduced modal coordinate which is transformed from DOF generalized coordinate $p_{s}$. $\Phi_{i}^{\prime}$ is

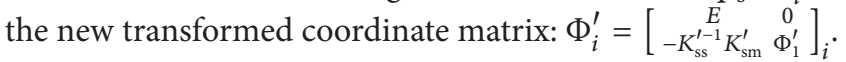
Here $\Phi_{1}^{\prime}$ is the low-order modal set with higher-order truncation. Thus, modal coordinate vector in (5) is transformed as $p_{i}=\left\{p_{\mathrm{m}}, p_{\mathrm{a}}\right\}_{i}^{\mathrm{T}}$.

To consider the rigid connection between substructures, the following coordinate transformation [12] is utilized to transform nonindependent coordinate $P=\left\{p_{1}^{\mathrm{T}}, p_{2}^{\mathrm{T}}, \ldots, p_{n}^{\mathrm{T}}\right\}^{\mathrm{T}}$ into the generalized coordinate of global structure.

$$
P=\beta z \text {. }
$$

Thus, the free vibration equation is established as follows:

$$
M \ddot{z}+K \dot{z}=0 .
$$

By the reduction of DOF, $M$ and $K$ are much less than mass matrix and stiffness matrix in the generalized coordinates of original system. Thus, natural frequency and modal shape can be obtained from (8).

2.2. Physical Model and Analysis Process. Blisk structure is a new manufacturing technology of aeroengine. The joint between tenon and mortise is removed out, so the weight of tenon-mortise connection and supporting structure is reduced greatly. In addition, bolts, nuts, locking plate, and other connectors are no longer needed. Compared with traditional blade disk, the hub becomes thinner in blisk structure, and bore diameter becomes larger. Figure 1 shows the structure of first two-stage blisk in aeroengine compressor. Number of blades in the first stage is 38 , and number of blades in the second stage is 53 .

In the finite element model of blisk structure, parts of blade and disk are established with the element Solid 185. As the blades on disk are leaned, the joint zone of blade and disk is meshed with the element Solid 187. Here total number of elements is 400124 and total number of nodes is 561878 . Blade material is titanium alloy TA11, the density is $4400 \mathrm{~kg} \cdot \mathrm{m}^{-3}$, elasticity modulus is $114 \mathrm{GPa}$, and Poisson's ratio is 0.3 . Disk material is titanium alloy TC17, the density is $8200 \mathrm{~kg} \cdot \mathrm{m}^{-3}$, elasticity modulus is $166 \mathrm{GPa}$, and Poisson's ratio is 0.3 .

For applying the prestressed CMS super-element method, substructure models of two-stage blisk are established in this research. The first-stage blisk and the second-stage blisk are packed as a substructure, respectively, as shown in Figure 2. In the first-stage substructure, it contains 194825 elements and 275406 nodes. In the second-stage substructure, it contains 205299 elements and 287432 nodes.

After establishing FEA model of substructure, the DOFs in hub tube are constrained for further analysis. Considering the effect of centrifugal force, operating rotational speed is applied. Prestressed analysis with fixed interface is performed in each substructure. Then, prestressed option is set as open; constrained master DOFs are released. Generation process of substructure mode synthesis is performed in the free interface, and super-elements are created. After these, application process is performed. The super-elements of substructures are connected to take the vibration analysis of whole model. Finally, dynamic response of super-element master DOFs is extended into each inner DOF of superelement orderly. Complete solution of dynamic response is obtained, and expansion process of the prestressed CMS method is accomplished. 


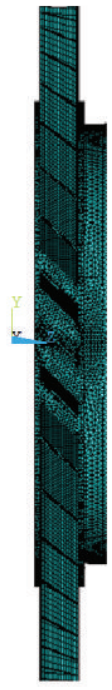

(a)

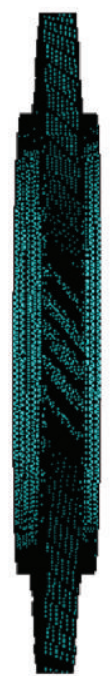

(b)

Figure 2: Substructure model of two-stage blisk: (a) the first-stage substructure and (b) the second-stage substructure.

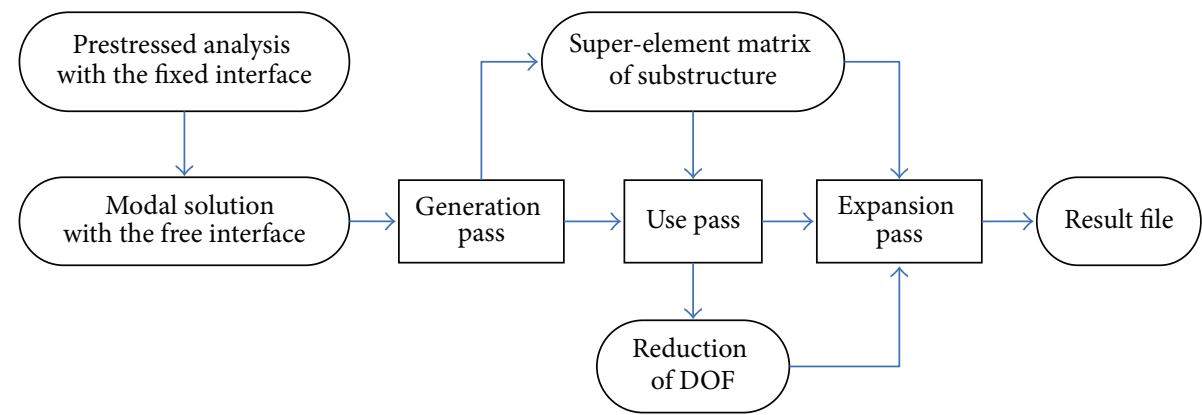

FIGURE 3: Analysis process of the prestressed CMS method.

Analysis process of prestressed CMS super-element method is shown in Figure 3.

\section{Vibration Characteristics of Blisk Structure}

3.1. Accuracy Verification of the Prestressed CMS Method. The modal truncation number is a key factor of calculation accuracy in prestressed CMS method. For obtaining more suitable modal truncation number in this research, natural vibration characteristics of blisk structure are calculated at different modal truncation numbers. Substructure models of two-stage blisk have been established, and operating speed of blisk structure is $11383 \mathrm{rpm}$. The DOFs of nodes in hub section are constrained as the boundary conditions. By the prestressed CMS method, natural frequencies of two-stage blisk are solved, respectively, at different modal truncation numbers. Here the result of global method is assumed as the accurate value. $N$ and $M$ are, respectively, the modal truncation number in the first-stage blisk and the secondstage blisk.

In Figure 4 , it is shown that calculation result of the prestressed CMS method at $M=35$ and $N=45$ is far away from the frequency of global method. With the increase of
TABLE 1: The comparison of computing time for two methods.

\begin{tabular}{lc}
\hline Type of method & Computing time/h \\
\hline$M=35, N=45$ & 3.7 \\
$M=40, N=55$ & 4.0 \\
$M=45, N=60$ & 5.1 \\
$M=60, N=75$ & 6.5 \\
Global method & 8.0 \\
\hline
\end{tabular}

$M$ and $N$, calculation results of the prestressed CMS method are much closer to the accurate solution. When $M=45$ and $N=60$, the result of prestressed CMS method is basically consistent with the result of global method. Continuing to increase the modal truncation number such as $M=60$ and $N=75$, calculation accuracy of prestressed CMS method is much higher. Therefore, it illustrates that the requirement of calculation precision can be satisfied.

For further examining the calculation efficiency of prestressed CMS method, computing time of prestressed CMS method is compared with that of global method at different modal truncation numbers, as is shown in Table 1 . 
TABLE 2: The comparison of natural frequencies at $M=45$ and $N=60$.

\begin{tabular}{lccc}
\hline Mode order & Global method/Hz & Prestressed CMS method/Hz & Vibration shape \\
\hline $1-10$ & $434.42-439.53$ & $434.45-439.58$ & Blade vibration of the first-stage blisk \\
$11-38$ & $440.36-442.86$ & $440.38-442.90$ & Blade vibration of the second-stage blisk \\
\hline 39 & 546.07 & 546.10 & \\
$40-43$ & $568.14-586.83$ & $568.21-586.90$ & $591.70-599.83$ \\
\hline
\end{tabular}

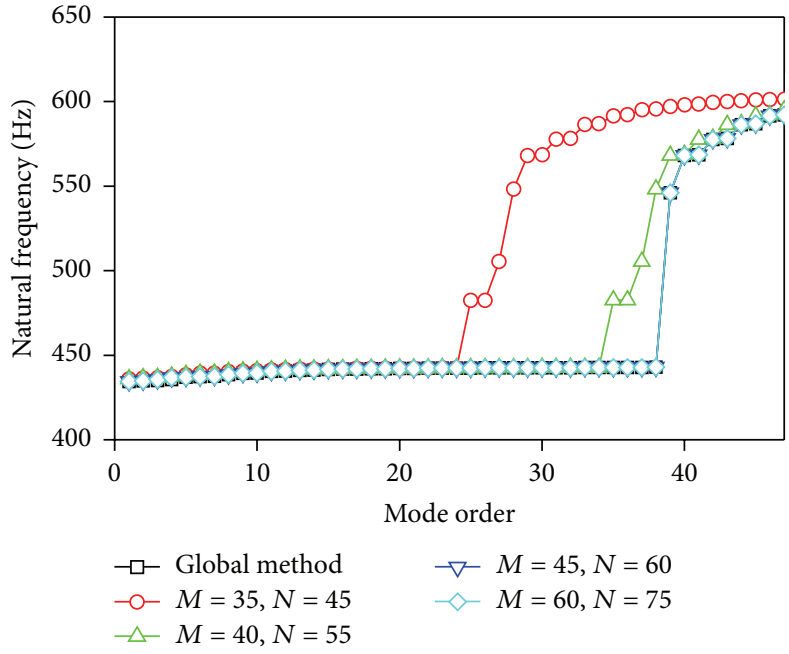

Figure 4: Natural frequency of blisk structure at different modal truncation numbers.

As shown in Table 1, computing time of prestressed CMS method increases with the growth of modal truncation number. When the modal truncation number rises to a certain value, computing time of prestressed CMS method is nearly equal to computing time of global method. It has been known that calculation accuracy of prestressed CMS method is improved significantly with the increasing of modal truncation number. While computing time is much longer, calculation efficiency of prestressed CMS method cannot be guaranteed. So the calculation accuracy and efficiency should be both considered to determine the modal truncation number.

From the above analysis, it can be known that prestressed CMS method has high enough precision at $M=45$ and $N=60$. Compared with the global method, calculation efficiency is improved by $36 \%$. For dynamic analysis of twostage blisk structure, hence $M=45$ and $N=60$ are selected as the modal truncation number. The results are compared with global method, as can be seen from Table 2.

Table 2 exhibits that 38 -order vibration frequencies of the first-stage blisk are in the range of $434 \mathrm{~Hz}-443 \mathrm{~Hz}$ and 53-order vibration frequencies of the second-stage blisk are in the range of $546 \mathrm{~Hz}-600 \mathrm{~Hz}$. It can be found that natural frequencies perform the bending shape of blade in loworder modes. The number of vibration modes is equal to the number of blades. The results of global method and prestressed CMS method have very small difference; it illustrates that prestressed CMS method is credible. In order to ensure the accuracy of prestressed CMS method, truncated frequency of the substructure is required to be greater than the corresponding frequency of solved system. For dynamic analysis of blisk structure, the modal truncation number is required to be greater than the number of blades. This selection principle of modal truncation number has been verified according to Figure 4 and Table 2.

3.2. Vibration Response of Two-Stage Blisk Structure. In the operation of blisk structure, main source of vibration is caused by the uneven air flow on blade pressure and suction surfaces. Airflow exciting force can be estimated on the basis of aerodynamic calculation, and the velocity and pressure on rotor blades can be determined by the experiment. Airflow exciting force at average radius of air passage can be expressed as the form of Fourier series; it is a series of harmonic superposition. For the system of blisk structure, practical exciting form is very complex; it has a great relationship with the working conditions. Assume that airflow exciting force on blade surface is simplified as the single point form. Aerodynamic force is applied at each blade tip of leading edge; the load in space is required to meet the form of traveling wave.

For the system of blisk structure, motion equation of forced vibration is as follows:

$$
M \ddot{x}+C \dot{x}+K x=f(t) .
$$

Here $x(t)$ is the vector of displacement and $f(t)$ is the vector of exciting force. The parameters $M, C$, and $K$ are mass matrix, viscous damping matrix, and stiffness matrix, respectively. In the forced response of blisk structure, exciting force $f(t)$ is usually expressed as

$$
f_{j}(t)=f_{j}^{0} \mathrm{e}^{i\left(\omega t+\phi_{j}\right)}, \quad j=1,2, \ldots, N .
$$

In formula (10), $f_{j}^{0}$ is the amplitude of exciting force in $j$ th blade, $\omega$ is the frequency of exciting force, and $N$ is the number of blades. $\phi_{j}$ is the phase of exciting force in $j$ th blade, and it is defined as

$$
\phi_{j}=\frac{2 \pi r(j-1)}{N}, \quad j=1,2, \ldots, N .
$$

Here $r$ is the order of exciting force.

3.2.1. Response Analysis of Vibration Displacement. Figure 5 shows the relationship between maximum displacement and 


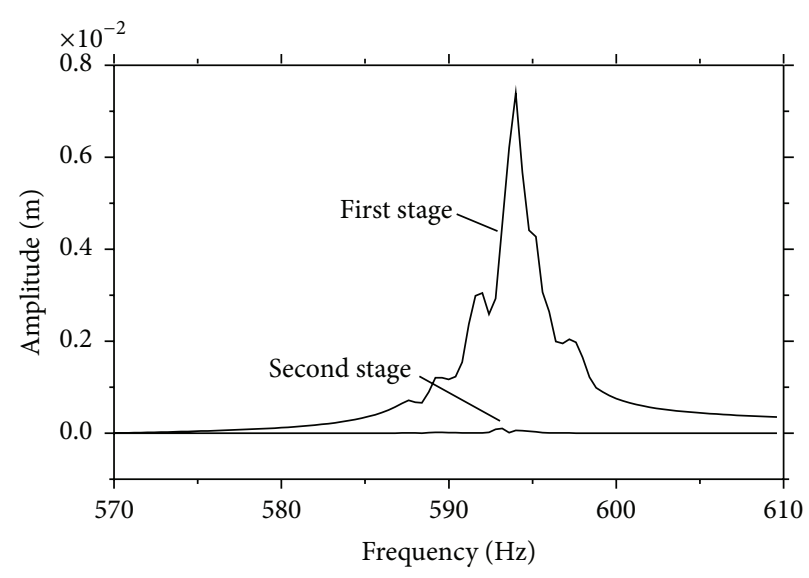

(a)

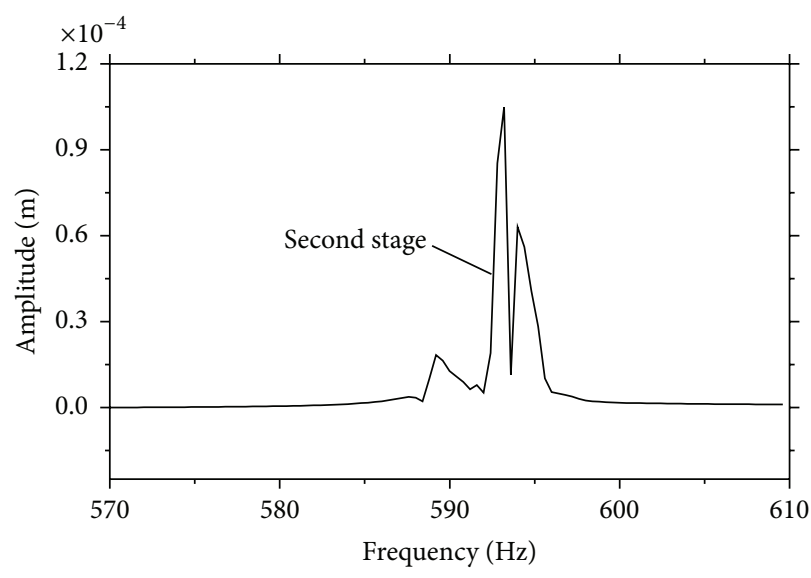

(b)

FIGURE 5: Comparison diagrams of displacement responses in two-stage blisk: (a) displacement responses of two-stage blisk and (b) enlarged view of the second-stage blisk.
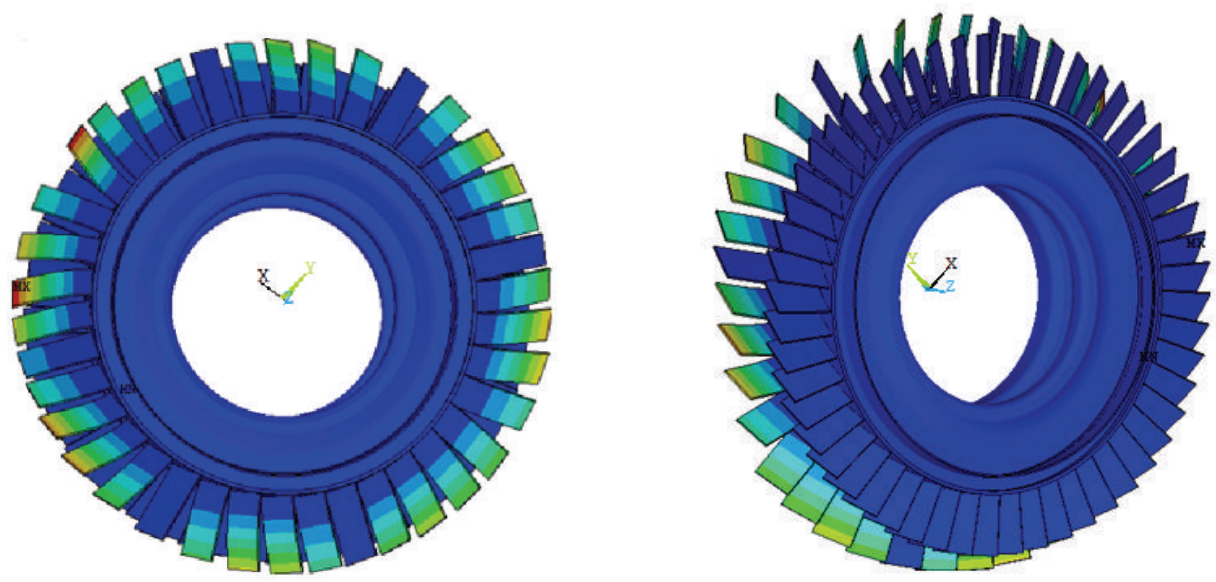

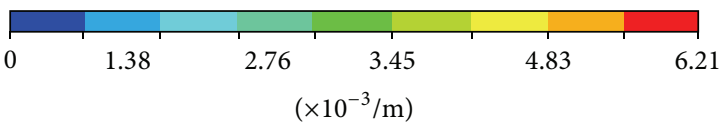

(a)

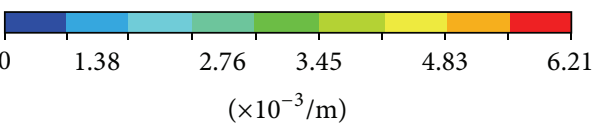

(b)

FIGURE 6: Displacement contour maps of two-stage blisk at resonant frequency $594 \mathrm{~Hz}$ : (a) front view of displacement contour map and (b) arbitrary view of displacement contour map.

exciting frequency, and the responses of vibration displacement in two-stage blisk are compared.

In Figure 5, it is exhibited that resonant frequency range of two-stage blisk is at $588 \mathrm{~Hz}-599 \mathrm{~Hz}$. The frequency is around $594 \mathrm{~Hz}$ at the maximum response. It is clear to note that the maximum displacement of the first-stage blisk is much higher than that of the second stage.

Figure 6 shows the displacement contour maps of twostage blisk at resonant frequency of $594 \mathrm{~Hz}$.

In Figure 6, it can be seen that when the frequency of exciting force is at $594 \mathrm{~Hz}$, the vibration of two-stage blisk is mainly focused on the first-stage blisk. Additionally, the maximum displacement appears at the position of blade tip, and the displacement vibration shows a nodal diameter mode. The amplitude of displacement vibration is relatively small in each region of the second-stage blisk.

3.2.2. Response Analysis of Dynamic Stress. The load acting on the element has a remarkable change with time, or each node on the component has significant acceleration under the load. The stress is generated by dynamic load in the component, and it is called dynamic stress. Dynamic stress analysis is the basis of solving the problem of component dynamic failure. According to the analysis of harmonic response, dynamic stress of two-stage blisk is obtained under aerodynamic excitation force. 


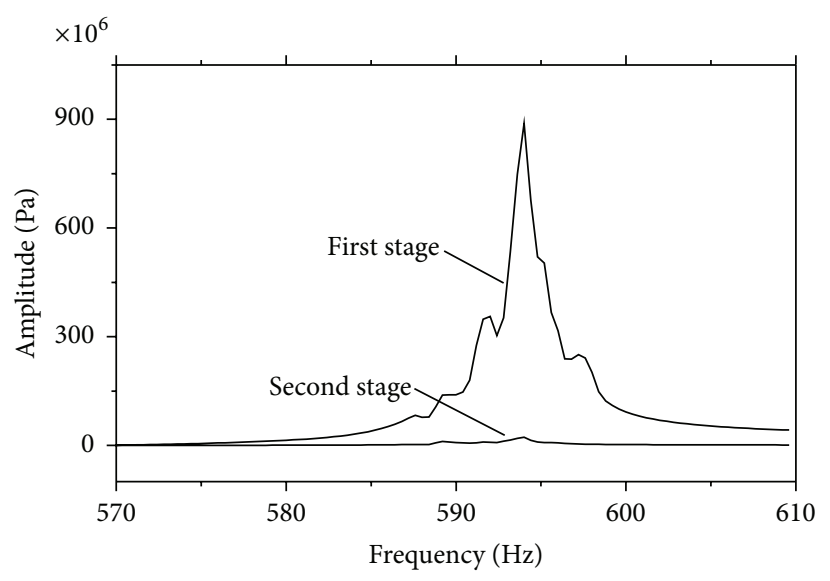

(a)

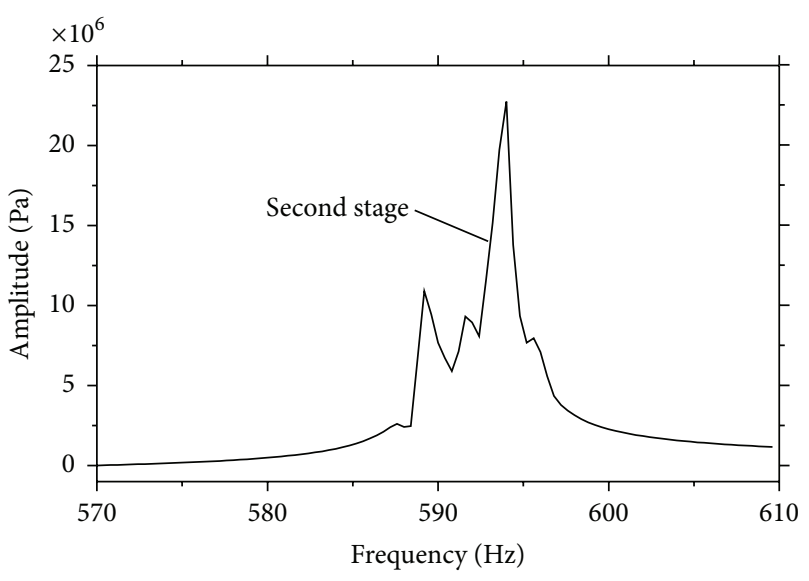

(b)

Figure 7: Comparison diagrams of dynamic stress in two-stage blisk: (a) stress responses of two-stage blisk and (b) enlarged view of the second-stage blisk.

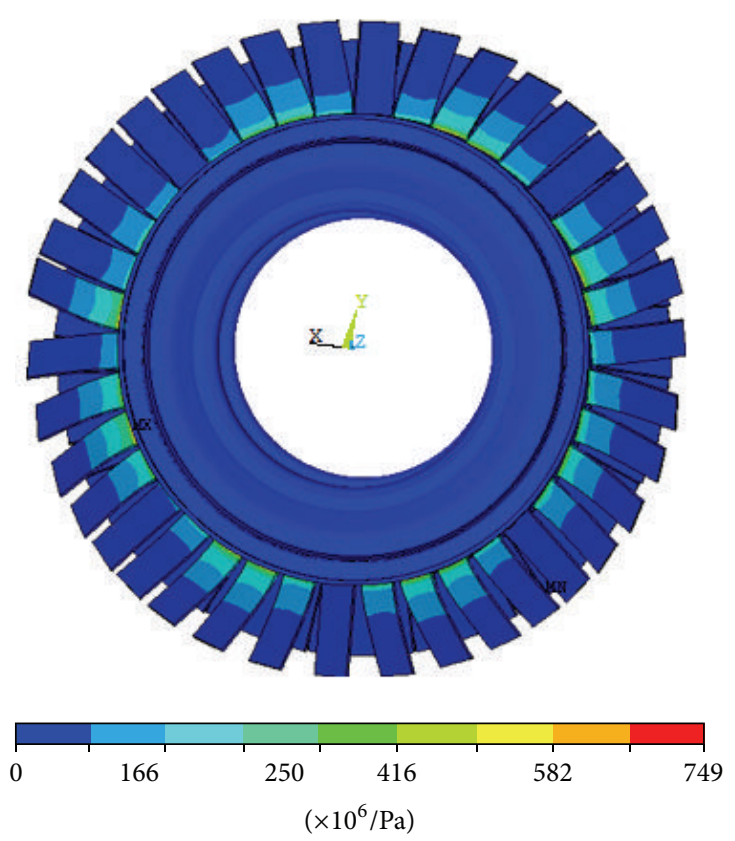

(a)
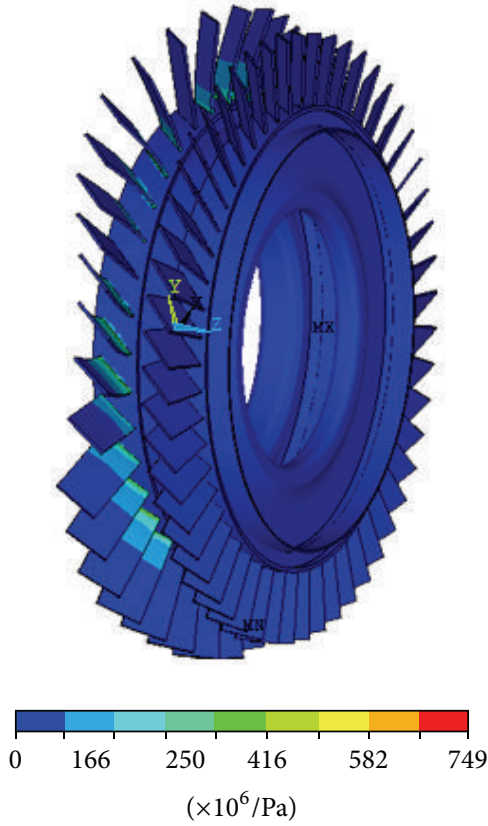

(b)

FIGURE 8: Dynamic stress contour maps of two-stage blisk at resonant frequency $594 \mathrm{~Hz}$ : (a) front view of stress contour map and (b) arbitrary view of stress contour map.

Figure 7 shows the relationship between maximum dynamic stress and exciting frequency; the responses of vibration stress in two-stage blisk are compared.

From Figure 7, it is found that the response of dynamic stress is basically consistent with the response of vibration displacement. The range of resonant frequency is at $588 \mathrm{~Hz}-$ $599 \mathrm{~Hz}$, and the response peak occurs at the frequency of $594 \mathrm{~Hz}$. Moreover, the maximum dynamic stress of the firststage blisk is much higher than that of the second-stage blisk.

Figure 8 shows the stress contour maps of two-stage blisk at resonant frequency of $594 \mathrm{~Hz}$.
In Figure 8, it can be shown that dynamic stress occurs mainly at blade root position of the first-stage blisk, and the stress vibration shows a nodal diameter mode. Besides, the stress amplitude of the second-stage blisk is relatively small in each region.

In order to avoid resonant response of blisk structure, the frequency of external load should be far away from the resonant frequency of $594 \mathrm{~Hz}$. In addition, it can be found that the maximum vibration displacement is $6.21 \mathrm{~mm}$ and the maximum dynamic stress is $749 \mathrm{MPa}$ in the limit working condition of $594 \mathrm{~Hz}$, while vibration amplitude and material 


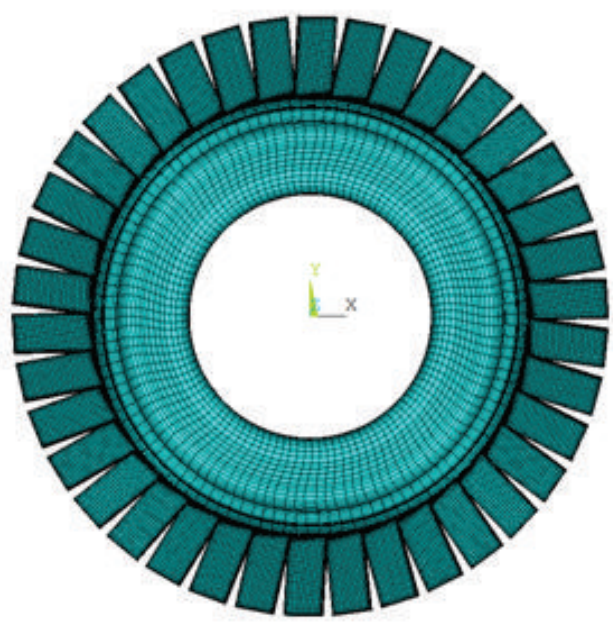

(a)

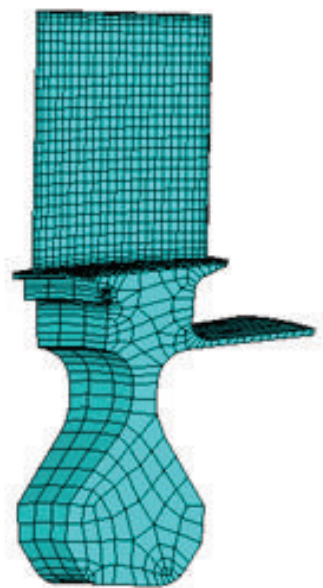

(b)

FIGURE 9: Models of global blisk and single-sector structure: (a) global blisk structure and (b) single-sector structure.

TABLE 3: Design of blade aspect ratios at fixed width condition.

\begin{tabular}{lccccc}
\hline Name & 1 & 2 & 3 & 4 & 5 \\
\hline Length/mm & 67.50 & 78.75 & 90.00 & 101.25 & 112.50 \\
Width/mm & 45.00 & 45.00 & 45.00 & 45.00 & 45.00 \\
Aspect ratio & 1.50 & 1.75 & 2.00 & 2.25 & 2.50 \\
\hline
\end{tabular}

strength of blisk structure are both in the safe working condition.

\section{Discussion of Blisk Vibration at Different Aspect Ratios}

Blade aspect ratio is the ratio of blade length and blade width; it stands for blade relative length or blade relative width. Blade aspect ratio is one of key factors on blisk vibration characteristics. In order to explore the effects of blade aspect ratios, natural frequencies of blisk structure are discussed at different aspect ratios.

4.1. Design and Modeling of Blisk Structure. For discussing the effects of aspect ratio on blisk vibration, blisk models are established at different aspect ratios. Relevant parameters including blade inclination and wheel size are kept constant in the process of modeling; blade aspect ratio is only adjusted accordingly. Thus, analysis results can be deemed to be credible.

According to practical experiences, blade aspect ratio $\lambda$ is set as $1.50,1.75,2.00,2.25$, and 2.50 , respectively, as shown in Tables 3 and 4. At the conditions of fixed width and fixed length, vibration characteristics of blisk structure are discussed.

After establishing blisk models at different aspect ratios, substructure models need to be divided. Substructure can be natural component of global structure, and it also can be a certain part of manual separation. As shown in Figure 9, blisk
TABLE 4: Design of blade aspect ratios at fixed length condition.

\begin{tabular}{lccccc}
\hline Name & 1 & 2 & 3 & 4 & 5 \\
\hline Length/mm & 90.00 & 90.00 & 90.00 & 90.00 & 90.00 \\
Width/mm & 60.00 & 51.43 & 45.00 & 40.00 & 36.00 \\
Aspect ratio & 1.50 & 1.75 & 2.00 & 2.25 & 2.50 \\
\hline
\end{tabular}

structure is divided into $N(N=38)$ sectors. Each sector of blisk is regarded as a substructure.

4.2. Blisk Vibration Characteristics at Different Aspect Ratios. Considering the effect of centrifugal force, blisk models at different aspect ratios are analyzed with the prestressed CMS super-element method. At the conditions of fixed width and fixed length, natural vibration frequencies of blisk structure are obtained. As blisk system has many blades, frequencies of the same vibration shapes are similar. Therefore, the first 120 order natural frequencies of blisk structure are solved. With the increase of blade aspect ratio, variation trends of natural frequencies and description of vibration shapes are illustrated as shown in Table 5.

For observing the relationship between natural frequencies and aspect ratios, one typical order of the similar frequencies is selected as the representative. The frequencies of 1st order, 39 th order, 77 th order, 84 th order, and 120 th order have been extracted as shown in Tables 6 and 7 .

According to the data in Tables 6 and 7, effect curves of aspect ratios on natural frequencies are drawn at the conditions of fixed width and fixed length, as shown in Figure 10.

From the analysis of effect curves in Figure 10, it can be found that each order frequency of blisk structure declines with the increasing of blade aspect ratio in the condition of fixed width. And the curve of high order frequency is much steep; it illustrates that the effect of blade aspect ratio on high order frequency is more obvious than the effect on low-order 
TABLE 5: Effect law of blisk natural frequencies with the increase of blade aspect ratio.

\begin{tabular}{|c|c|c|c|}
\hline Mode order & Fixed width & Fixed length & Description of vibration shape \\
\hline 1 & $\searrow$ & $\searrow$ & Radial expansion of blade and wheel \\
\hline $2-38$ & $\searrow$ & - & First-order bending vibration of blade \\
\hline 39 & $\searrow$ & $\searrow$ & First-order blade bending with 0 nodal diameter \\
\hline $40-77$ & $\searrow$ & $\nearrow$ & Distorted vibration of blade \\
\hline 78 & - & - & Wheel vibration with 0 nodal diameter \\
\hline $79-120$ & $\searrow$ & $\searrow$ & Blade bending vibration with nodal diameter \\
\hline
\end{tabular}

“-" stands for no obvious change, “ $\nearrow$ " stands for rise, and " $\searrow$ ” stands for decline.

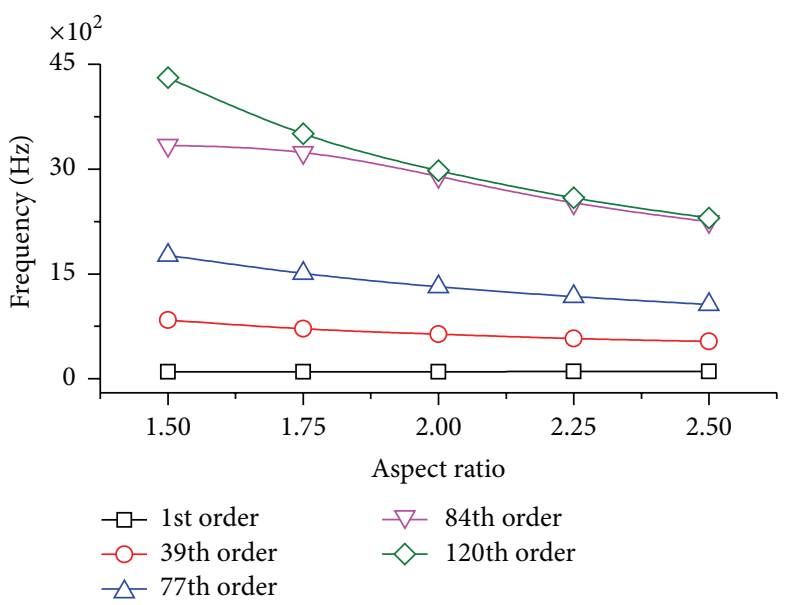

(a)

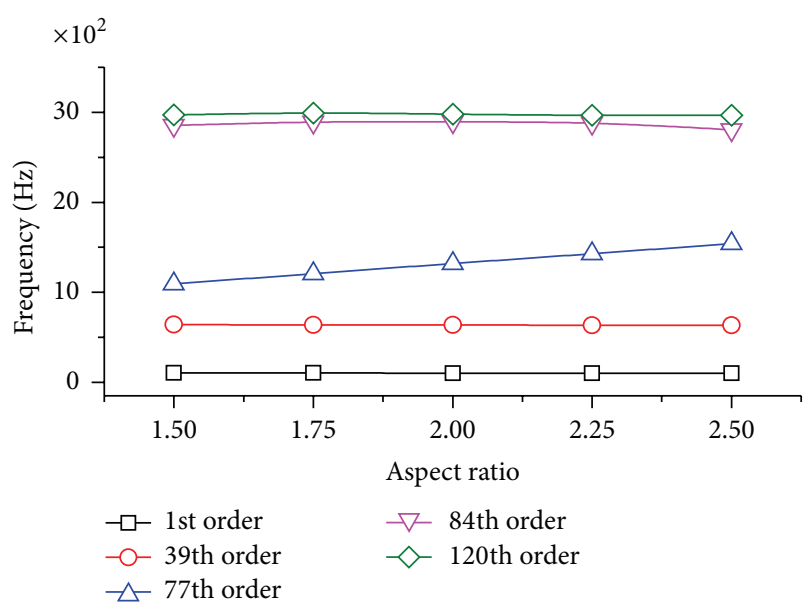

(b)

FIGURE 10: Effect curves of natural frequencies at different aspect ratios: (a) condition of fixed width and (b) condition of fixed length.

TABLE 6: Natural frequencies of typical orders at different aspect ratios of fixed width.

\begin{tabular}{lccccc}
\hline $\begin{array}{l}\text { Aspect } \\
\text { ratio }\end{array}$ & 1st & 39th & 77th & 84th & 120th \\
\hline 1.5 & 99.19 & 839.86 & 1769 & 3341 & 4307 \\
1.75 & 100.35 & 717.73 & 1508 & 3239 & 3506 \\
2 & 101.66 & 636.4 & 1319 & 2895 & 2979 \\
2.25 & 103.14 & 578.26 & 1176 & 2520 & 2590 \\
2.5 & 104.4 & 534.58 & 1062 & 2250 & 2300 \\
\hline
\end{tabular}

TABLE 7: Natural frequencies of typical orders at different aspect ratios of fixed length.

\begin{tabular}{lccccc}
\hline $\begin{array}{l}\text { Aspect } \\
\text { ratio }\end{array}$ & 1st & 39th & 77 th & 84 th & 120th \\
\hline 1.5 & 103.51 & 640.82 & 1094 & 2854 & 2971 \\
1.75 & 102.52 & 638.58 & 1208 & 2892 & 2993 \\
2 & 101.66 & 636.4 & 1319 & 2895 & 2979 \\
2.25 & 101.14 & 634.69 & 1430 & 2878 & 2969 \\
2.5 & 100.63 & 632.64 & 1540 & 2804 & 2966 \\
\hline
\end{tabular}

frequency. In the condition of fixed length, blade distorted frequencies between 40 th order and 77 th order show a certain rise with the increasing of blade aspect ratio. However, other order frequencies have no clear change.

\section{Conclusions}

In this research a prestressed CMS super-element method is put forward for the vibration analysis of aeroengine blisk structure. Based on this method, dynamic characteristics of blisk structure are calculated at different modal truncation numbers. And the effects of different blade aspect ratios have been discussed on blisk vibration characteristics. Through the above analysis, we can draw a conclusion.

(1) Compared with the result of global method, the accuracy of prestressed CMS method can meet the requirement of blisk dynamic analysis. For the selection principle of modal truncation number, natural frequency of the substructure is required to be greater than the corresponding frequency of solved system.

(2) Resonant frequencies of the first-stage blisk and the second-stage blisk are basically consistent; they are mainly at $588 \mathrm{~Hz}-599 \mathrm{~Hz}$. The maximum displacement and maximum dynamic stress appear at blade tip and blade root of the first-stage blisk, respectively, and show the vibration mode of nodal diameter.

(3) Effects of aspect ratio on blisk vibration are different at the conditions of fixed width and fixed length. 
Natural frequencies of blisk structure decline with the increasing of blade aspect ratio in the condition of fixed width, and the effect of blade aspect ratio is more obvious on high order frequency, while blade distorted frequencies show a certain rise in the condition of fixed length.

\section{Competing Interests}

The authors declare that they have no competing interests.

\section{Acknowledgments}

The work is supported by the National Science Foundation of China (Grant no. 51275081) and the National Science Foundation of China (Grant no. 51335003).

\section{References}

[1] H. Ferria, P. Ferrand, F. Pacull, and S. Aubert, "Numerical investigation of flutter stability in subsonic space turbine blisk with emphasis on cut-on/cut-off modes and interblade phase angles," Journal of Thermal Science, vol. 21, no. 6, pp. 492-499, 2012.

[2] D. Di Maio and D. J. Ewins, "Experimental measurements of out-of-plane vibrations of a simple blisk design using blade tip timing and scanning LDV measurement methods," Mechanical Systems and Signal Processing, vol. 28, pp. 517-527, 2012.

[3] J. Ji, D. Zhang, S. Li, and B. Chen, "A master model approach to multidisciplinary design and simulation for compressor blisk," Advanced Materials Research, vol. 308-310, pp. 209-212, 2011.

[4] S. K. Bhaumik, T. A. Bhaskaran, R. Rangaraju, M. A. Venkataswamy, M. A. Parameswara, and R. V. Krishnan, "Failure of turbine rotor blisk of an aircraft engine," Engineering Failure Analysis, vol. 9, no. 3, pp. 287-301, 2002.

[5] S. Lu and F.-J. Lu, "Structure optimization design for blisk based on ANSYS," Journal of Aerospace Power, vol. 27, no. 6, pp. 12181224, 2012.

[6] F. Xu, C.-S. Li, Q.-S. Luo, B.-H. Zhou, and C.-B. Wu, "Study on the blade crack of an axial flow compressor blisk," Gas Turbine Experiment and Research, vol. 25, no. 4, pp. 25-29, 2012.

[7] Y. G. Tsuei and E. K. L. Yee, "Direct component modal synthesis technique for system dynamic analysis," Journal of Biological Chemistry, vol. 171, no. 2, pp. 639-640, 1947.

[8] T. Mochizuki and I. Hagiwara, "A comparison between modal differential sub-structure method and conventional component modal synthesis methods," Journal of System Design \& Dynamics, vol. 5, no. 2, pp. 320-331, 2011.

[9] J.-B. Qiu, Z.-G. Ying, and L. H. Yam, "New modal synthesis technique using mixed modes," AIAA Journal, vol. 35, no. 12, pp. 1869-1875, 1997.

[10] P. Ren, L. Wei, and G. Gong, "Analysis for modal characteristics of crane boom based on modal synthesis method," Hoisting \& Conveying Machinery, vol. 7, pp. 59-64, 2014.

[11] W. C. Hurty, "Vibrations of structural systems by component mode synthesis," Journal of the Engineering Mechanics, vol. 86, pp. 51-70, 2015.

[12] M. C. C. Bampton and R. R. Craig Jr., "Coupling of substructures for dynamic analyses," AIAA Journal, vol. 6, no. 7, pp. 13131319, 1968.
[13] S. N. Hou, "Review of modal synthesis techniques and a new approach," Shock and Vibration Bulletin, vol. 40, no. 4, pp. 2539, 1969.

[14] R. L. Goldman, "Vibration analysis by dynamic partitioning," AIAA Journal, vol. 7, no. 6, pp. 1152-1154, 1969.

[15] R. H. MacNeal, "A hybrid method of component mode synthesis," Computers \& Structures, vol. 1, no. 4, pp. 581-601, 1971.

[16] S. Rubin, "Improved component-mode representation for structural dynamic analysis," AIAA Journal, vol. 13, no. 8, pp. 995-1006, 1975.

[17] W. L. Wang and Z. R. Du, Structural Vibration and Substructuring Method, Fudan University Press, Shanghai, China, 1985.

[18] Y. T. Leung, "An accurate method of dynamic substructuring with simplified computation," International Journal for Numerical Methods in Engineering, vol. 14, no. 8, pp. 1241-1256, 1979.

[19] W. Yun, G. Duan, and Z. Hu, "The superelement method of modal synthesis and its application to dynamlc calculation of ship structure," Journal of Shanghai Scientific Research Institute of Shipping, no. 1, pp. 1-18, 1981. 


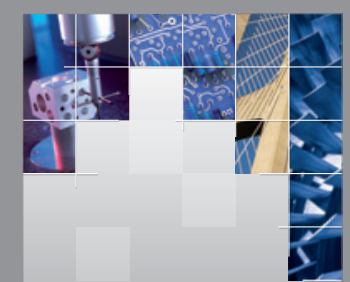

\section{Enfincering}
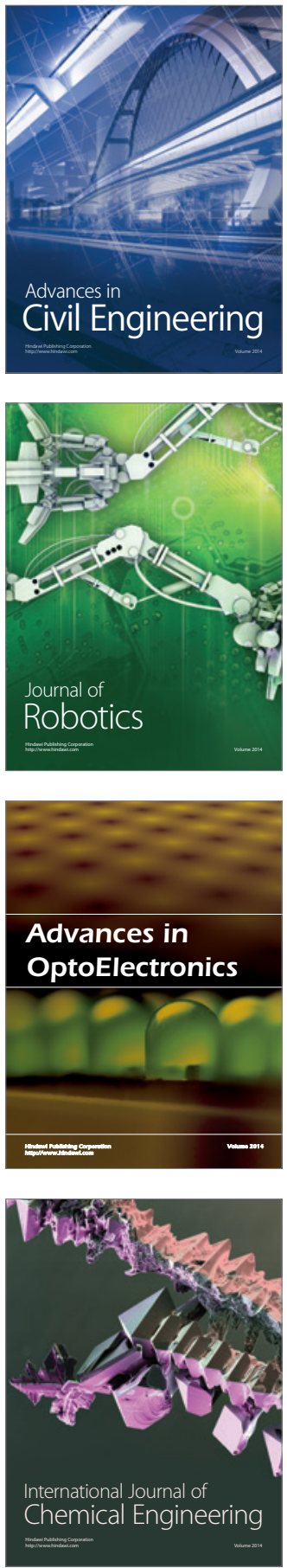

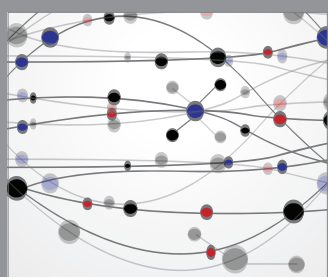

The Scientific World Journal

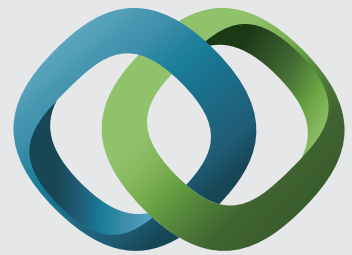

\section{Hindawi}

Submit your manuscripts at

http://www.hindawi.com
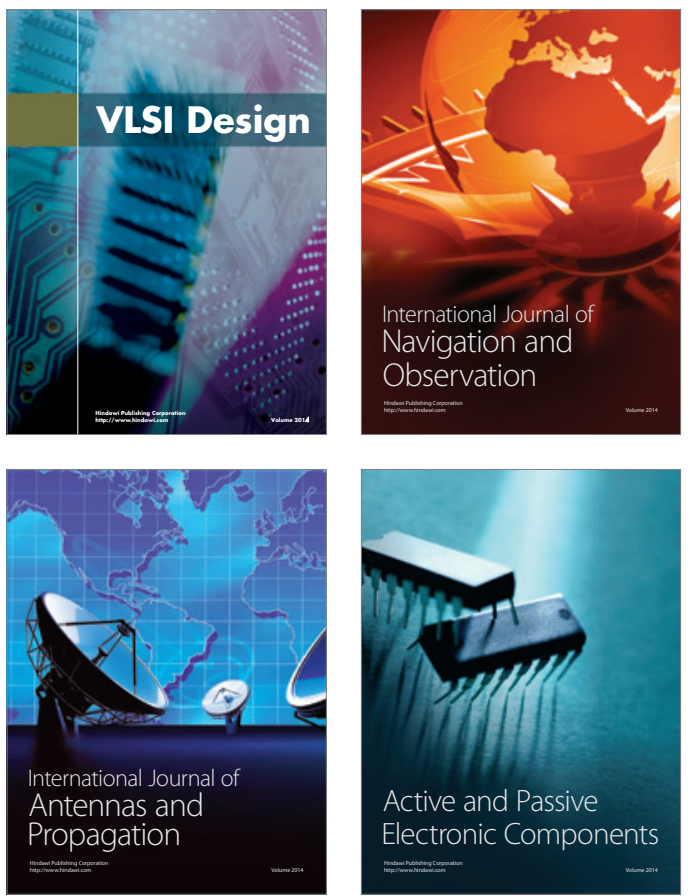
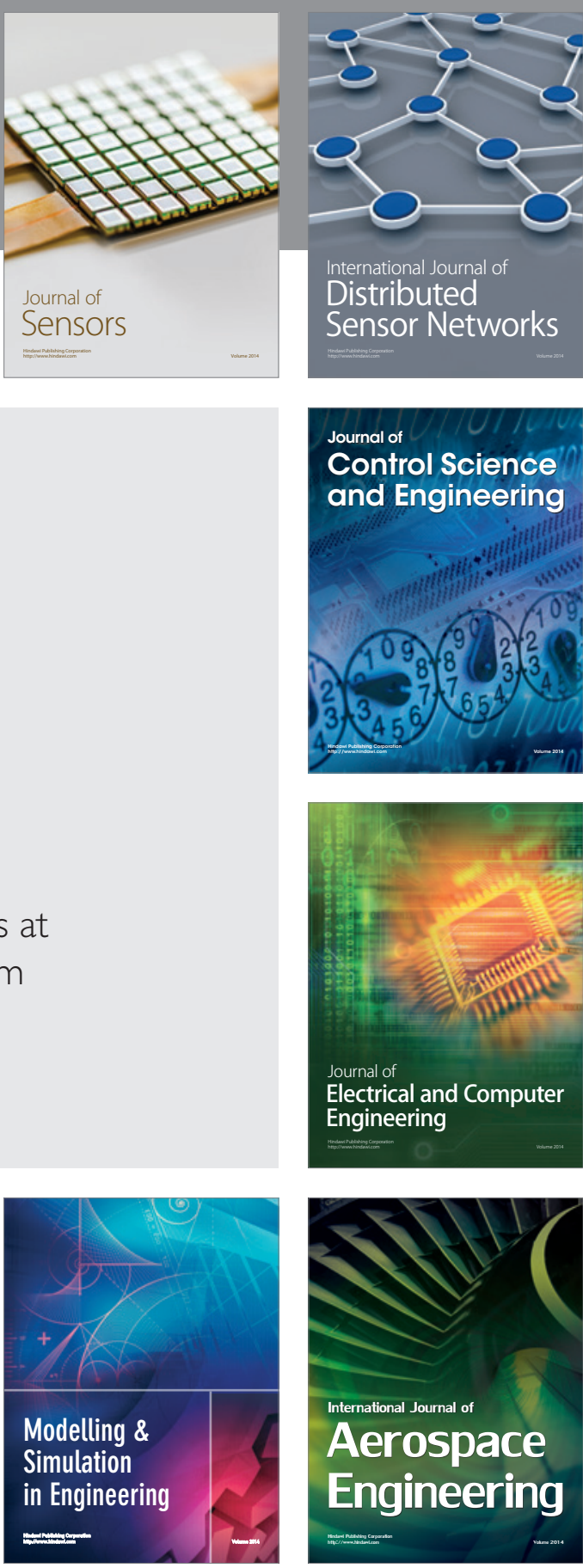

International Journal of

Distributed

Sensor Networks

Journal of

Control Science

and Engineering
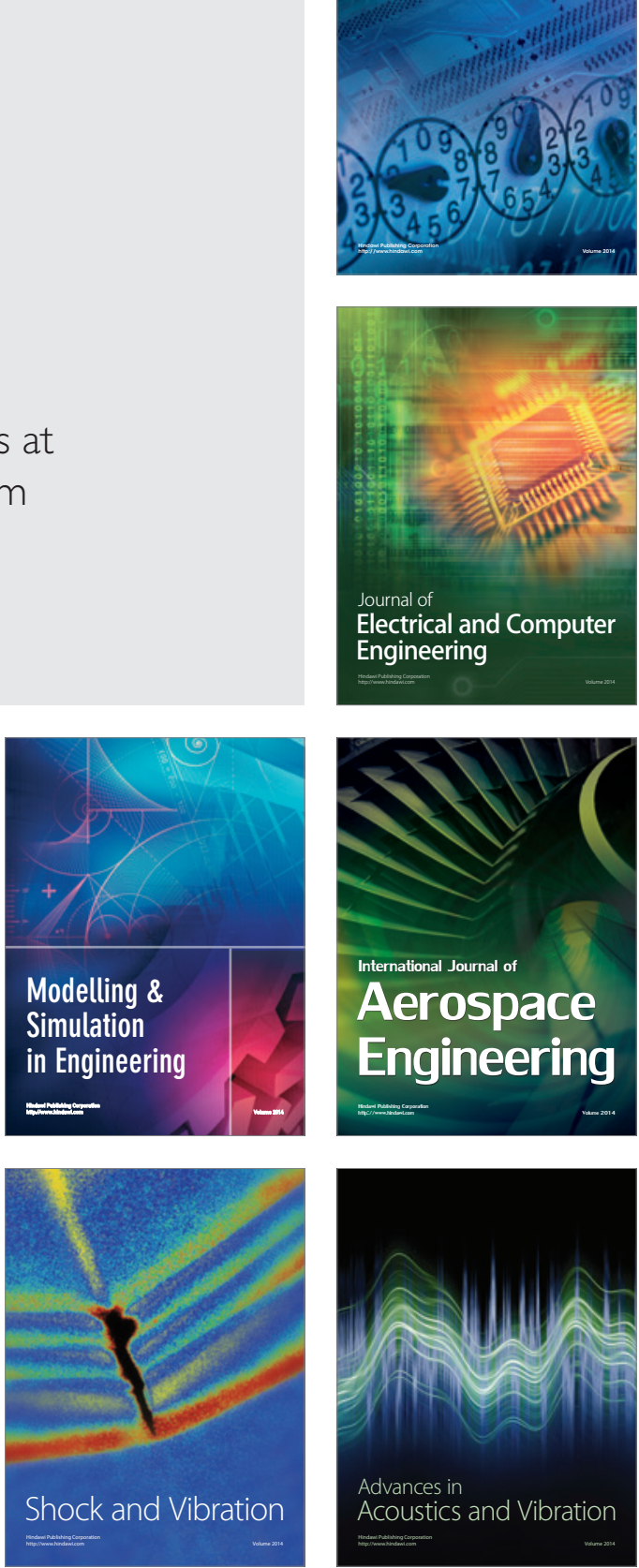